\title{
The Added Value of Multislice Computerized Tomography in Evaluation of Maxillofacial Fractures
}

\author{
Mohammad Tharwat Mahmoud Solyman ${ }^{1}$, Ahmed Mohammed Abd El Menam \\ Gafer $^{2}$, Salma Ahmed Ragheb ${ }^{1 *}$ \\ Departments of ${ }^{1}$ Radiodiagnosis and ${ }^{2}$ General Surgery, Faculty of Medicine- Sohag \\ University, Egypt
}

\begin{abstract}
Background: As Regard fracture morphology, The maxillofacial fractures are often complex, so the imaging findings should be familiar to the clinicians. To diagnose maxillofacial fractures, several radiographic approaches have been utilized. Recently, Multidetector computed tomography (MDCT) with three-dimensional (3D) and multiplanar reformation (MPR) become basic in fractures evaluation because of its higher

sensitivity.

Objective: The study purpose to 1- To study the number, extent, and degree of fractures displacement through the utilization of axial, coronal \& 3D reformatted images. 2. Study the value of axial, coronal \& 3D reformatted images in fractures recognition.

Methodology: This study has been done through the collection of data from papers which has been searched upon PubMed from 2001 till 2017, the search is done including only the papers written in English language and collected data including maxillofacial region anatomy, a hint about the CT physics and injury presentation. Summary:The fracture is classified into orbital, facial and mandibular fractures and the facial fractures are sub-classified into upper, mid and lower face fractures. In complex midface fractured patients, it easy detects frontal and maxillary bones fractures and their displacement. In comparison with X-ray, Mandibular CT imaging, in particular, MDCT, It is highly sensitive in mandibular fracture detection, and provide excellent detail in detecting mandibular condyle fractures. and The coronal reconstruction has a superior role in the orbital and maxillary fractures detection. Conclusion: MDCT with (3D)and (MPR) images become a standard piece of fractures evaluation due to sensitivity of this imaging system for fracture.
\end{abstract}

Keywords: MDCT, Evaluation Of Maxillofacial Fractures, CT

\section{INTRODUCTION}

The first imaging modality in trauma patients is Plain radiography, yet its significance has been declined because of deficient data in surveying the seriousness of the damage in maxillofacial injury. Although there is a risk of radiation, computed tomography (CT) scan defeating the issue of the superimposition of structures that definitely happens on plain radiographs as it has the advantage of giving pictures of thin slices of the facial skeleton. Furthermore, electronic manipulation of the data has been allowed by the digital acquisition of information through altering the settings to provide images either at bony window levels or for efficient soft tissue evaluation in contrast to the analog information of plain films [1].

MDCT helps detection of the definitive number, exact site and extent of fractures, soft tissue damage, and displaced fragments and in much less time. Fractures of the face are categorized into mandibular and midface fractures. There may be isolated 
bone fractures or complex facial fractures [2].

Taking into consideration the anatomical sites. the radiological investigation is strongly indicated in severe facial trauma [3]. In an acute trauma setting, care must be given to ensure the airway is secured and associated injuries related to the patient's primary survey are excluded before the fracture is assessed, suspected mandibular fracture if a patient presents with malocclusion, broken teeth, trismus [4].

\section{AIM OF THE WORK}

The motivation behind the investigation to:

1- To study the number, displacement, and extent of fractures with axial, coronal \& 3D reformatted images.

2. Study the efficacy of axial, coronal $\&$ three-dimensional (3D) CT reformatted images in recognition of maxillofacial fractures.

\section{ANATOMY OF}

\section{MAXILLOFACIAL REGION}

The maxillofacial region can be separated into three sections:

- The upper face - the frontal bone and frontal sinus within.

- The midface - the nasal, ethmoid, zygomatic, maxillary bones and maxillary sinus within.

- The lower face - the mandible [5].

The region of the orbit:

- The frontal bone forms a superior orbital margin.

- The frontal process of the greater wing of the sphenoid bone, the zygoma, and the zygomatic process of the frontal bone form the lateral margin of the orbit.

- Zygomatic as well as Maxillary bones from the inferior margin of the orbit.

- The medial orbital margin formed by the lacrimal bone, ethmoid bone, frontal process of the maxillary bone, the angular process of the frontal bone and orbital process of the frontal bone as well.

- The roof of the maxillary sinus forms the floor of the orbit.

- Parts of the palatine, sphenoid and ethmoid bones form the apex of the orbit [4].

Blood and sensory supply:

- Blood supply of the face arising from branches of the external carotid artery.

- The motor supply for muscles responsible for facial expression is derived from the facial nerve.

- The ophthalmic, maxillary and mandibular branches of the trigeminal nerve supply sensation to the skin of the face [6].

An incomparable diagnostic approach when dealing with an unidentified entity (pathologic or not) has been obtainable by Multiplanar imaging that has stood as a diagnostic experiment. This idea is inherent to the volumetric type of data (computed tomography [CT], cone beam computed tomography [CBCT], magnetic resonance imaging) and the exclusive facility in the direction to make images (sections) at different planes (flat or curved) has been offered to the diagnostician. Because of the ability of CBCT to acquire and store a volume of data, this data can be realigned or be reformatted and the diagnostician can synthesize several various types of images in any way requires, along these lines superimposition of the territory or entity under investigation has been disposed with other neighboring structures and its assessment has been permitted from all points of view. With multiplanar imaging, there-createtion of the images in various planes (flat or curved) has been done with very humble utilities, accordingly expanding the diagnostic accuracy in the hands of the knowledgeable individual in a matchless way (Fig. 1) [7]. 
Unquestionably, cone-beam imaging provides multiplanar imaging, which is unique for the dental expert: most dentists and authorities, with just a couple of exemptions, are inexperienced with diagnostic imaging in various planes, in spite of the fact that keening on projection images analysis as those created by traditional dental imaging modalities (intraoral radiography as well as panoramic radiography). The spatial relationship of the various known anatomic structures in the maxillofacial area has been revealed by sectional images (tomographic images), which was pretty much lost in projection imaging [7].
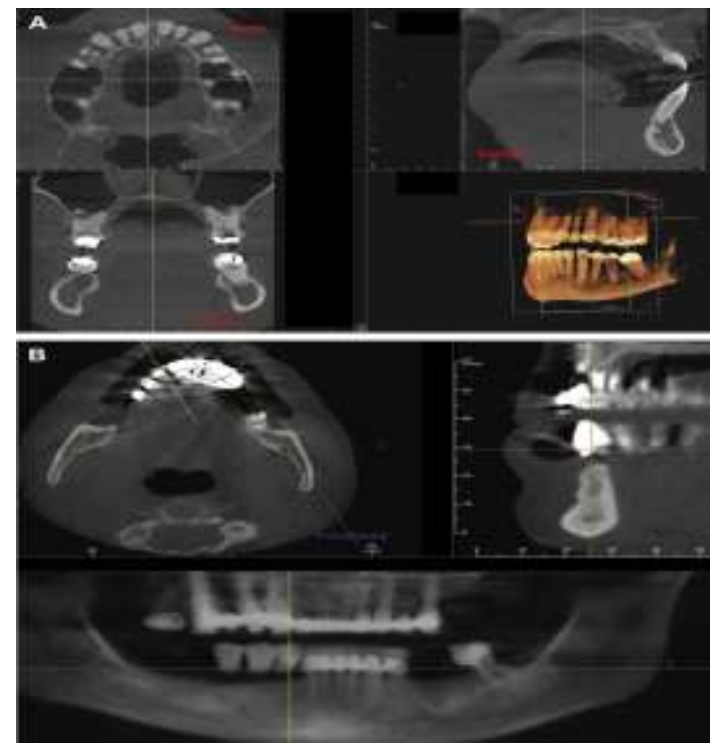

Fig. 1: Multiplanar imaging [7].

\section{MAXILLOFACIAL} FRACTURES

\section{Types of maxillofacial Fractures:}

There are numerous reasons causing facial fractures, which can incorporate, interpersonal trauma, motor car accidents (MCA), sportsrelated activities or occupationalrelated accidents. Patients are frequently gotten in the emergency room for primary assessment. In the primary management period, lethal problems are first to deal with and facial fracture repairment only endeavors after patient's condition stabilization. Nearly all patients get a fine cut (thin slice thickness) computed tomography (CT) scan for evaluation of the skeletal trauma. Occasionally if suspect a jaw fracture a panorex or dental $\mathrm{x}$-ray is recommended [8].

\section{Orbital fractures:}

Orbital fractures can happen secluded or associated with more severe facial injuries. direct injury from the front such as trauma from a ball or a fist often results in these fractures.

Common symptoms include the following:

1. Bruising and swelling and around the eye

2. Blurring of vision or seeing double

3. Cheek Numbness just below the injured eye

4. Sunken look to the eyeball

Surgical intervention is not intended for all orbital fractures. The surgeon is more likely to recommend surgery if:

1) visualization compromisation or 2) Sunken appearance of the eye, as a result, sagging of eyeball into the socket (which may not be clear at first a result of swelling) [9].

\section{Ethmoid-nasal complex fractures:}

Likewise, with other facial fractures, Ethmoido-nasal complex fractures, or fractures of the sinus bones between both eyes (Ethmoid sinus), can happen with other facial trauma. High-velocity trauma usually results in these fractures.

Common symptoms include:

1. Severe swelling of the orbit

2. Periocular bruising

3. Widening appearance around the eyes [10].

\section{Maxillary fractures:}

Fractured maxillary bone fractures that involve the region of the midface. High-speed trauma, i.e. motor vehicle accidents usually the consequence of these fractures. 
1. Common symptoms include the following:

2. Unfitted teeth together properly or Change in dental occlusion

3. Problems in vision

4. Nasal drainage of Clear watery fluid The objective of surgical intervention for maxillary fractures is to reestablish the ordinary shape of the facial skeleton and insurance that a patient's bite functions or jaw are as ordinary as would be prudent. Numerous modalities can be utilized for assessment the fractures and may incorporate incisions through gums or the mouth or in more widespread fractures, incisions in the hairline. once fractured bones are moved back into place and balanced out, Utilization of plates and shield the bones from sliding again[11].

Fractured Mandible bone:

Mandible or jawbone fractures are the second common fracture in line in facial trauma (the most widely recognized being nasal fractures). Frequently the mandibular fractures are not only in one fractured site but two.

Common symptoms include the following:

1. Pain and tenderness of the jaw

2. Improper ability to bring teeth together

3. Underneath tongue bruising

4. Chin numbness

7-10 days after the injury is the ideal time to repair jaw fractures. In some cases, the doctor will endorse antibiotics to take before the surgery if the fracture extention extended inside the mouth area[12].

Radiologic imaging of maxillofacial fractures:

Conventional X-rays: In the late 1940 s and 1950 s various radiographic techniques were account for the appraisal of dentoalveolar, midfacial, mandibular, and pan facial injuries. Mandibular and condylar fractures were identified as the utilization of a combination of lateral-oblique [13].

Dentoalveolar injuries: The chief imaging technique in dental trauma evaluation still is the Intraoral radiography. While the most common recognized presentation of dental trauma is the clinical crown fractures, itemized data about potential root fractures and the displacement of teeth have been provided with the periapical radiograph and peri-operatively the correct situating of an avulsed or luxated tooth into the alveolus can be confirmed[14].

Mandibular fracture: The plain x-ray skull radiography is frequently enhanced with rotational panoramic radiography used in a patient with mandibular fractures. A higher degree of diagnostic accuracy in distinguishing mandibular fractures has been provided through it [15]. In one study, the panoramic radiograph (Fig. 2) shows that it could diagnose $92 \%$ of the mandibular [16].

\section{Midface fractures:}

Midface fractures can be divided into:

1-Central midface fracture: It incorporates nasal, naso-orbitoethmoid (NOE) fractures, isolated maxillary fractures and the three types of Le- Fort fractures. The classical pure form of Le- fort fractures is uncommonly observed.

2-Lateral midface fracture: It includes the zygomatic complex fractures (trimalar or tripod fracture) which is the second commonest facial fracture and less common zygomaticomaxillary fractures, zygomaticomandibular fractures, and isolated zygomatic arch fracture. Nasal bone Fractures are considered being the commonest fractures in the maxillofacial region. Clinical diagnosis or plain radiographs could assess them 
adequately while extensive $\mathrm{NOE}$ fracture requires CT [16].

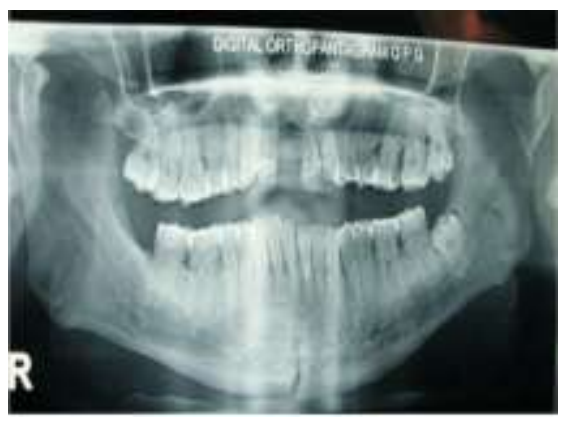

Fig. 2: OPG having a fracture of the symphysis and bilateral condylar fracture of the mandible [16].

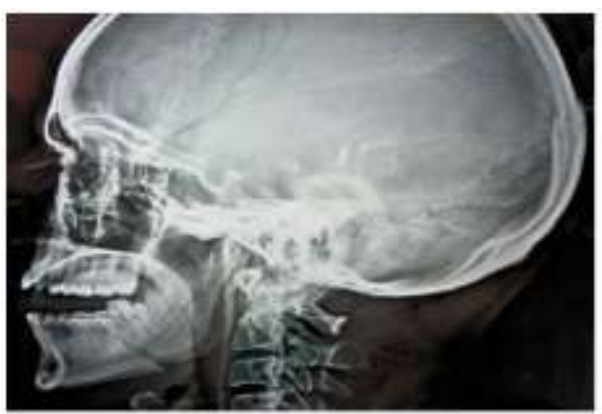

Fig. 3: Lateral view of the face showing a fracture of the body of the mandible [16].

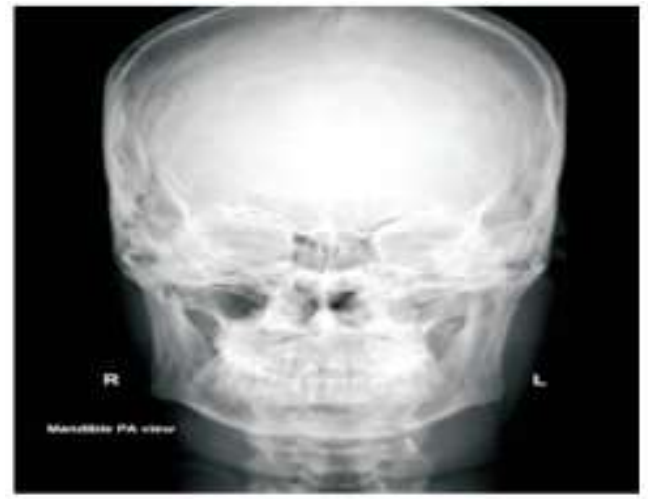

Fig. 4: PA views of mandible showing a fracture of Lt. body of the mandible [16].

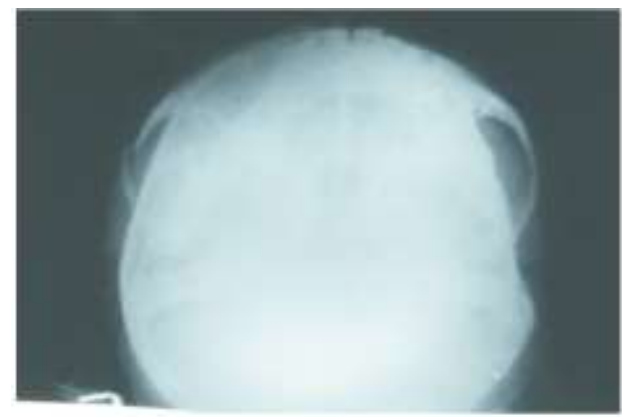

Fig. 5: Submento-vertical view showing a fracture of Lt. zygomatic $\operatorname{arch}[16]$.

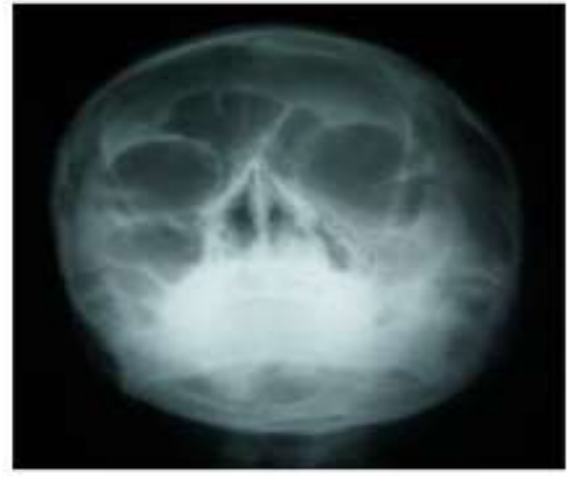

Fig. 6: Occipitomental view radiograph showing zygomatic complex fractures [16].

Magnetic resonance imaging (MRI): MRI is an imaging strategy that utilization radio-waves instead of $\mathrm{X}$ rays, MR scanning is substantially less effective in imaging bone than $\mathrm{CT}$ and is more time consuming than CT. MRI is the ideal procedure in the assessment of Temporomandibular joint pathology. Its outstanding soft-tissue contrast resolution makes it perfect for internal derangement of the joint detection, and it can be used to show synovitis, joint effusion, erosions, and associated bone marrow edema. It has an adjunctive role to $\mathrm{CT}$ in the assessment of orbital soft tissues and in particular blow out fractures. It can be utilized to look for cerebrospinal fluid (CSF) leak after skull base injuries as well. An uncommon bad outcome of skull base trauma is a carotico-cavernous sinus fistula. In this case, MRI and MR angiography are helpful in making a diagnosis [17].

\section{CT IN DIAGNOSIS OF MAXILLOFACIAL FRACTURES \\ Computed tomography (CT)} examination, known as computerized axial tomography (CAT) examination as well, is a diagnostic imaging procedure based upon the idea of 
utilization $\mathrm{x}$-rays to build crosssectional images ("slices") of the body. Cross-sections are reconstructed from estimations of attenuation coefficients of $\mathrm{x}$-ray beams in the volume of the object studied [18].

The essential guideline of the CT is based on that the density of the tissue passed by the $\mathrm{x}$-ray beam can be estimated from figuring the attenuation coefficient. Using this principle, The density of the body reconstruction is allowed by the CT, by twodimensional (2D) section opposite to the axis of the acquisition system [18]. Basically speaking there are two procedures of the absorption: Compton and photoelectric impacts. A solitary coefficient, mju represents this phenomenon. In certain, CT cases, the X-rays emitter rotates around the patient and the detector, placed in the entirely opposite side, in order to get the picture of a body section (beam and detector move in synchrony) [19].

Dissimilar x-ray radiography, no images are produced by the detectors of the CT scanner. The measurement of the transmission of a thin beam (1$10 \mathrm{~mm}$ ) of $\mathrm{x}$-rays can be done through a full diagnostic CT examination of the body. The image of that section is taken from various angles, and this permits to collect the data on the depth (in the third dimension) [19].

The Computerized Tomography imaging utilization in the nuclear medicine field has been growing, first with Duke combined with CAT (PET/ Computerized

Tomography) introduction and, all of the more as of late, with SPECT combined with CT (SPECT/CT) presentation. A portion of hidden CT thoughts have been included, such as projection reconstruction (i.e., estimating information at numerous positions and angles), has a high law of similarity to those basic the cross-sectional nuclear medicine imaging modalities PET and SPECT [20].

\section{Constrains of ordinary radiography}

Screen radiography has several defaults that make its capacity to picture low-balanced tissues and structures with adequate radiation exposure levels of patient restricted. These impediments incorporate the following [21].

Inefficient x-Ray Absorption

High Scatter-to-Primary x-Ray Ratios

Conspicuity and Superimposition

Receptor Contrast Versus Latitude

Fundamental principles of CT: the original CT (the first generation computerized tomography)

The theme to be inspected has been imagined by Hounsfield imagined being split into axial sections. He collimated the used x-ray beam down to a thin (pencil-width) $\mathrm{x}$-ray beams. The size of the beam was $13 \mathrm{~mm}$ wide opposite to the segments (along the axis of the subject) and $3 \mathrm{~mm}$ inside the plane of the cut. In fact, the cut thickness to be filtered has been normally controlled by the beamwidth. On the other side of the subject, the $\mathrm{x}$ ray tube is fixed to an $\mathrm{x}$-ray detector located strictly. Together, the tube and the detector scan across the subject, scanning the thin $\mathrm{x}$-ray beam through the slice. A translation implies this transverse examination of the detector and the tube over the subject. The arrangement is diagramed in Figure 7[22].

During translation motion, the detector estimates the transmission of the $\mathrm{x}$-ray beams through the subject. A ray has been defined as the $\mathrm{x}$-ray beam passes through the subject related to every measurement. A view is characterized as a gathering of estimations that were made during the translation and their associated beams. Hounsfield's Mark I scanner estimated the transmission of 160 rays for every 
view. The relating number of estimations for today's scanners is ordinary over 750 . After complete, the translation process, Around the subject, the tube-detector is turned by 1 , and so as to accomplish the subsequent view, the translation process is to be repeated. If the event that the primary translation process is accomplished with the detector underneath the subject and the tube over (0), at this point the subsequent translation is accomplished with the tube-detector get together at 1 . This process has to be repeated by the Mark I scanner in 1 increment in order to accomplish 180 views over $180.1,000$ or more views over 360 Today's scanners might be typically collected by today's scanners (the purpose behind the substitution of 180 views by 360 views will be clarified later). The gathering of incremental rotation which follows the linear translation is called translaterotate motion. Gathering of the data was acquired with single sodium iodide (NaI) scintillation detector and a single thin beam. First-generation CT geometry is the prementioned arrangement (single narrow beam and single detector with translate-rotate motion) and it requires 5-6 $\mathrm{min}$ so as to finish a scan. To decreases the examination time, In order to collect the data for 2 slices at the same time, 2 adjoining detectors and a 26-mm-wide $\mathrm{x}$-ray beam (in the slice thickness direction) actually have been used by Mark I scanner. By scan has been ended, 28,800 measurements (180 views · 160 rays) for each slice has been delivered by Hounsfield, those taken at many positions (160) and angles (180) [22].

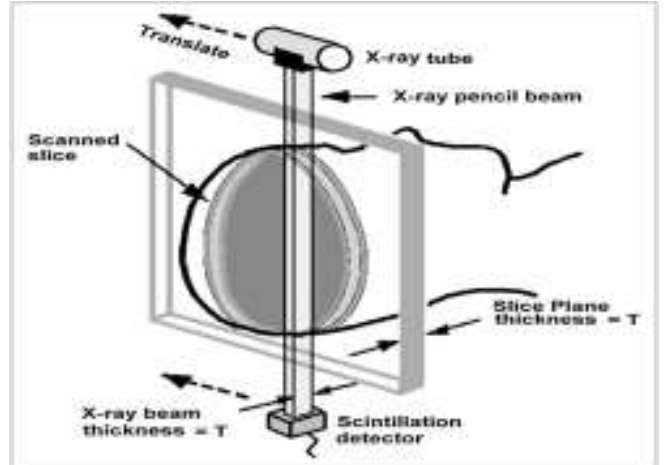

Fig. 7: CT course of action. Sweeping of the axial slice through patient by narrow (pencil-width) $\mathrm{x}$-ray beam as linked $\mathrm{x}$-ray tube-detector apparatus examines over a patient in linear translation. Translations are to be repeated at Various angles. Slice thickness defined as the thickness of a thin beam [22].

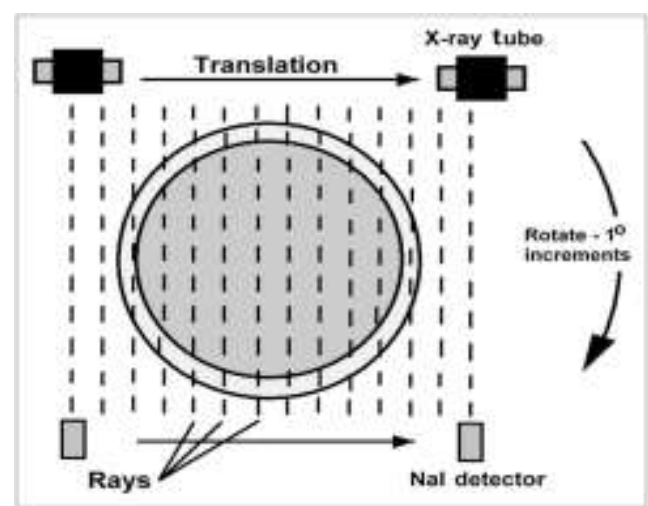

Fig. 8: The Measurements of transmission of X-ray. Estimations are gained at various focuses through translation motion of tube and detector. A ray refers to the X-ray path which corresponds to every measurement, and a view refers to a group of rays which have been measured through translation. In order to obtain data that is sufficient for reconstruction of images, Views are collected at many angles (in 1 increase in this example) [22].

\section{CT image presentation}

From the most punctual long stretches of a CT a convention that was existed is that attenuation value calculated for every voxel of the matrix which was reconstructed is currently 
being replaced with an integer (CT number) determined as follows:

$$
\begin{aligned}
& \text { CT number (in Hounsfield units, HU) } \\
& \quad=\left[\mathrm{K} \times\left(\mathrm{u}_{\text {qoxel }}-\mathrm{u}_{\text {waker }}\right)\right] / \mathrm{u}_{\text {wiler }} . \quad \text { Eq. } 2
\end{aligned}
$$

In this equation, uvoxel is the calculated voxel attenuation coefficient, uwater is the attenuation coefficient of the water, and $\mathrm{K}$ is an integer steady value. In the new EMI scanner, $\mathrm{K}$ becomes standardized as 1,000 (or sometimes 1,024 ), in the original one $\mathrm{K}$ was 500 . In the next section, the explanation behind $\mathrm{CT}$ numbers computation with respect to water is talked about. Today scanners determine uwater from waterequivalent phantoms or periodic calibration scans of water. Because of $\mathrm{x}$-ray beam energy influences attenuation coefficients, appropriate $\mathrm{x}$ ray generator calibration adjustment is significant for reproducible and accurate $\mathrm{CT}$ numbers. Note that the CT number is the quantity, However, the units are Hounsfield units (to respect the designer). In this way, for instance, It would be said that a particular tissue in a picture "has a CT number of 40 Hounsfield units." [22].

\section{The original EMI CT scanner}

The first EMI scanner was a dedicated head scanner wherein the patient's head was recessed by means of an elastic film into a water-filled box (additional Figs. 9 and 10). The gadget was wanted to such a degree, that the water-filled box rotated (in 1 increase) alongside the singular-thinbeam, single-detector get together, bringing a fixed way through the patient notwithstanding to water for all beams and transmission estimations. The outcomes achieved with this first restoratively based EMI scanner (introduced in September 1971) were exhibited at a British radiologic culture meeting in April 1972. The outcomes left no vulnerability as to the progressive clinical estimation of the procedure. The noteworthy accomplishment of the scanner made various intrigue and prompted the impact of innovative work by numerous gatherings and companies. one such advancement was FBP reconstruction, depicted prior. $\mathrm{Be}$ that as it may, a technology race to improve and grow the CT procedure was likewise in progress. Obviously, body scanning, is one of the significant applications, yet the water-filled box had to be dispensed with, in any case, is advantageous. It filled 2 needs, the two of which permitted Hounsfield to boost the exactness of attenuation coefficient estimations [23].

\section{Beam-Hardening Correction}

$\mathrm{X}$-rays made in $\mathrm{X}$-ray cylinders are for the most part Bremsstrahlung $\mathrm{x}$ rays which, dissimilar the discrete photon energies created by radioactive isotopes, spread an immense continuum of energies (up to a most outrageous numerically proportionate to the $\mathrm{x}$-ray tube kilovoltage). Such beams allude to as polychromatic. Beam hardening alludes to a dynamic increase in the undeniable energy of polychromatic $\mathrm{X}$-ray beams as they enter further into attenuating materials. It is realized about by special attenuation of the lower-energy (and therefore less penetrating) photons in the beam by each successive layer of attenuating material [24]. Since attenuation coefficients rely on both the material and beam energy, a comparable tissue at a more noteworthy profundity has a lower attenuation coefficient (in the light of that the deeper tissue is, the lesser the $\mathrm{x}$-ray beam hardening attenuates). During checking of a uniform object (e.g., a cylindric water-filled phantom), beam hardening causes lower attenuation coefficients to be 
reproduced for profound voxels, making an undesired "cupping", artifact in which comparative material seems darker in the point of the convergence of the picture than in the fringe. To get seeing how the waterfilled box allowed Hounsfield to address for this beam-hardening effect, consider the method of one of the estimations through the water-filled box and patient. As in Equation 1, the estimations are expressed as communicated as a whole of the attenuation coefficients of the voxels through which it passes [24]:

$$
\mathrm{Xa}=\mathrm{u} 1+\mathrm{u} 2+\mathrm{u} 3+\mathrm{u} 4+\ldots . . .:+\mathrm{un}:
$$

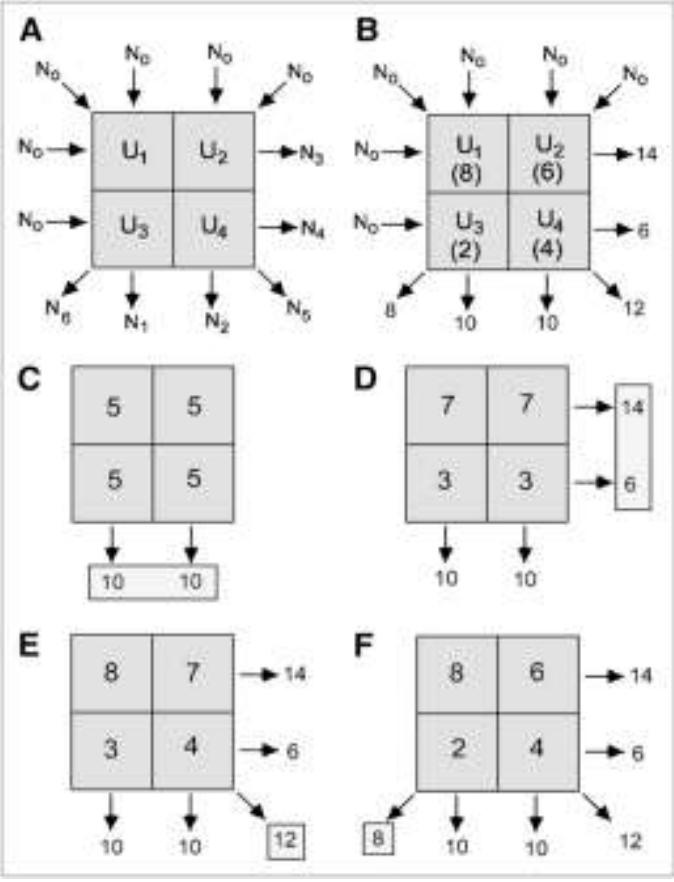

Fig. 9: ART. (A) ART calculation for 4-voxel "patient." (B) Attenuation estimation. (C) Starting assessment is worked by isolating estimations from principal view comparable along with their beam ways. (D-F) This estimate is iteratively acclimated to match measurements for each successive view, stopping when transmission estimations predicted by current estimate match all actual measurements to within some preset resilience [23].

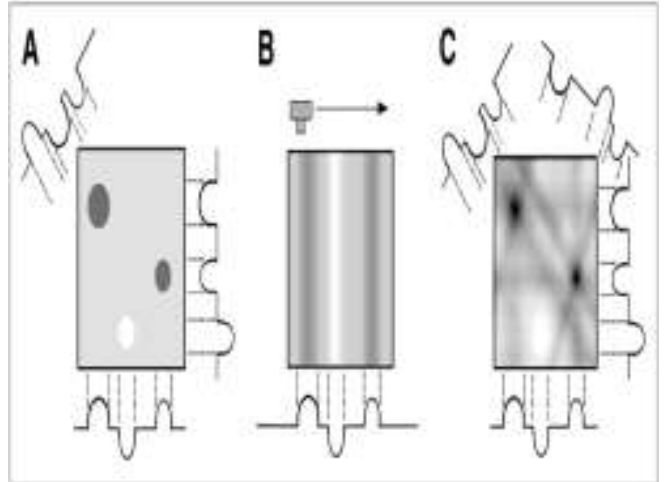

Fig. 10: (A) Backprojection reconstruction for straight forward apparition containing 3 objects with various attenuation values. (B) For each view, attenuation values are just separated equitably along with their beam ways. Summing back-projected views from variable angles build an image. (C) Four views of the phantom are summed. In spite of the fact that this strategy is productive, images reconstructed with back-projection shows extensive haziness[23].

\section{ROLE OF CT IN DIAGNOSIS OF MAXILLOFACIAL FRACTURES}

Fracture morphology of maxillofacial injuries are frequently intricate, so the clinicians ought to be acquainted with the imaging discoveries. Distinctive radiographic strategies have been utilized so as to identify maxillofacial trauma. Panoramic imaging is being used broadly so as to screen of orofacial trauma as well as other diseases. Cone-beam computed tomography (CBCT) is additionally utilized in order to diagnose orofacial ailments [25]. Nonetheless, regardless of a higher dose of radiation in correlation with the plain radiography, in craniomaxillofacial injuries, CT is the imaging procedure of choice pass judgment on various fragments, the pivot and separation degree, or any skull base association [26].

High-resolution multiplanar reformation (MPR) and isotropic survey are allowed by multidetector computed tomography (MDCT) 
permits; all of which improve the indicative intensity of this imaging modality, Hence profiting maxillofacial trauma patients, and can distinguish the non-displaced fractures and besides give three-dimensional (3D) morphology of the more complex fractures in maxillofacial trauma [27]. As of late, MDCT with MPR and 3D images have turned into a fundamental piece of the appraisal of facial accidental injury in the light of awesome affectability of this imaging strategy for fracture [28].

\section{Mandibular fractures}

CT was more sensitive than panoramic tomography, particularly for fractures of the angle, condyle, or mandibular ramus [29]. Condylar fractures have been perceived in $64.8 \%$ of all patients with mandibular fractures using MDCT [28]. For various investigations, $48.0 \%$ of patients with fractures of the mandible had condylar fractures using radiographic assessment [30], and condylar fractures accounted for $50.1 \%$ of the mandibular fractures utilizing panoramic radiography and CT examinations [31]. We consider the instability of condylar fractures utilizing MDCT were higher than those of different reports in light of the exquisite sensitivity of MDCT. Mandibular fractures were gathered by the dissemination of the scattering delineated by Lieger et al. [32] into four types: median, paramedian, angle and condylar types.

The most outstanding mandibular fracture site was the condyle (33.6\%), trailed by the angle (21.7\%), and various mandibular fractures were found in $48.6 \%$ of patients [33]. With respect to mandibular fractures appropriation, the dominant $(25.0 \%)$ occurred in the condyle and $23.0 \%$ in the angle [34]. The most frequently involved mandibular fracture sites are
The condyle (38.2\%) and the median (27.0\%) [35].

In all mandibular fracture patients, the fracture lines were various in $44.4 \%$ [35]. The condylar type was most common (47.1\%), followed by the median type $(20.9 \%)$, and in all mandibular fracture patients, the percentage of numerous fractures were $50.7 \%$ [28]. No difference of the percentage in fractures of the mandible between multiple and single fractures has been suggested by these reports. Statistically, Both bilateral and unilateral condylar are associated with the median-type fractures [31]. 75.2\% was the percentage of cases with a condylar fracture within whole multiple mandibular fracture percentage, $66.7 \%$ of median mandibular fracture type, $45.5 \%$ of paramedian mandibular fracture type, and $12.3 \%$ of angle of the mandible fracture type. It has been considered that indirect fractures of the condyle with or without fractures in the median region caused by trauma force were applied in the median region. Fig. 11 shows the MDCT of mandibular fractures [28].

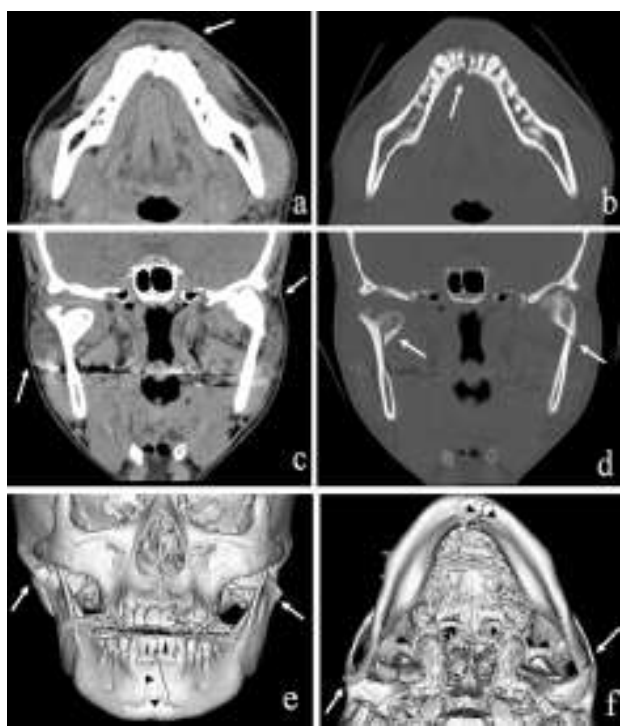

Fig. 11: A 46-year-old male with mandibular fracture coming about on account of falls. Axial image (a) exhibits a median fracture accompanied by soft-tissue edema 
(arrow). Axial image (b) exhibits a median fracture (arrow). Coronal image (c) exhibits condylar fractures accompanied by soft-tissue edema (arrows). Coronal image (d) exhibits condylar fractures (arrows). 3D images ( $\mathrm{e}$ and $\mathrm{f}$ ) to better exhibits show median (arrowheads) and condylar fractures (arrows) [28].

\section{Midfacial fractures containing maxillary fractures}

There are few kinds of midfacial fractures, including Le Fort I, II, and III fractures, foremost maxillary fractures, zygomaticomaxillary complex fractures and others [36]. Sohns et al. [36] showed that orbital fractures $(22 \%)$, maxillary fractures (21\%), nasal bone (14\%), and zygomatic bone $(9 \%)$ were the most witnessed fractures seen in their study. Smith et al. [37] indicated that nasal bones (19\%), malar and maxillary $(28 \%)$, and orbital (41\%) were common fractures. These reports propose the distinction of rate in midfacial fractures, notwithstanding the orbital fractures were by far the most of the fractures are seen. In the midface, in falling injuries, the zygomaticomaxillary complex fracture was most frequently involved [38]. In falling accidents, the most widely recognized fracture of facial trauma was the zygomaticomaxillary complex. The most commonly observed zygomaticomaxillary complex fractures were due to violence. A hit with a fist is by far the most comm0n type of relational viciousness, yet highenergy implies, for example, the utilization of kicking or, knuckles reinforcement is expanding [27]. We think that the high-velocity coming about because of falls or brutality was applied in the facial region, causing direct fractures of the zygomaticomaxillary complex type. Figure 12 demonstrates the MDCT of zygomaticomaxillary complex fractures. Maxillofacial bones support functions such as seeing smelling, breathing, eating, and speaking. Therefore, maxillofacial fractures necessitate that radiologic analysis to be accurate to prevent severe functional cosmetic deformity and debilities through using MDCT and surgical management [37].

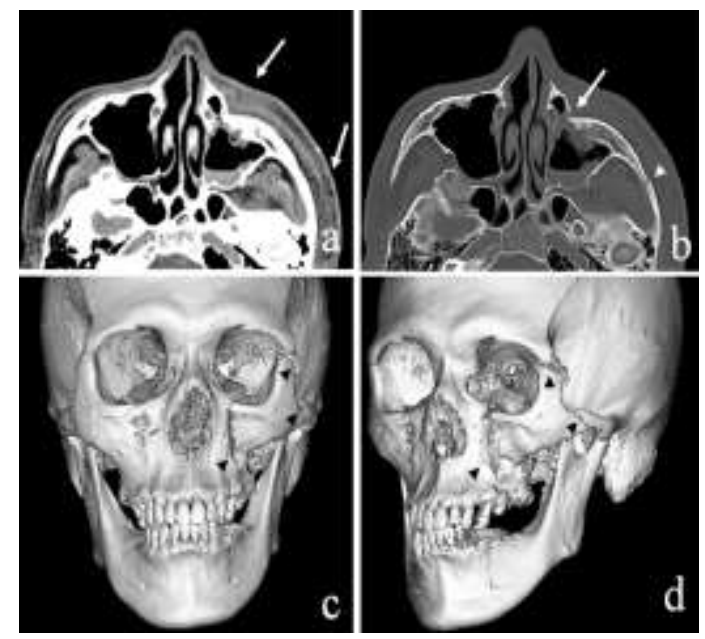

Fig. 12: A 45-year-old male with midfacial fractures resulting in from falling injury. Axial image (a) exhibits zygomatic arch and maxillary fractures accompanied by soft tissue edema (arrows). Axial image (b) exhibits zygomatic arch (arrowhead) and maxillary (arrow) fractures. 3D images (c and d) to a better demonstration, It shows zygomaticomaxillary complex fractures (arrowheads) [37].

Fractures of the temporal bone accompanying with mandibular trauma

Injuries involving different zones of the cranial and maxillofacial skeleton are frequently accompanying the temporal bone fractures. Complications of fractures of temporal bone incorporate sensorineural and conductive hearing loss, dural sinus thrombosis, facial nerve injury, carotid dissection, orofacial pain with unidentified complaint and cerebrospinal fluid leakage [39]. Therefore, For early trauma cares it is necessary to make a diagnosis of 
fracture of temporal bone early. Temporal bone fractures after mandibular trauma are thought to be an uncommon element, and just a couple of case reports have depicted fractures of the tympanic plate and the glenoid fossa of the temporal bone accompanying with mandibular trauma [40]. The tympanic plate and glenoid fossa fracture of the temporal bone may happen when the posterior bony wall is being impacted by the fractured or unfractured mandibular condyle. As clinicians are commonly in charge of every symptomatic finding When MDCT is being performed [41].

\section{The radiation dose of MDCT}

As to radiation dose of $\mathrm{CT}$, the actual dose for the maxillomandibular volume imaging with CT examination methods is altogether higher than that with CBCT [41], and it has been described with fractures of the mandible [42]. However, trauma encompassed loss of consciousness as well as Ambulatory patients with a suspected facial fracture. In order to effectively detect, locate, figure the degree of fragment dislocation degree of fragment dislocation, soft-tissue edema, and hemorrhage. The MDCT is being considered as an effective device. So, It is recommended replacing CBCT with the MDCT, especially for patients who presented with extensive craniomaxillofacial trauma, loss of consciousness and impaired vital functions [40].

\section{SUMMARY}

The advantages of CT and 3D images include assessment of accuracy and extension of fracture in the maxillofacial region. In complex midface fractured patients, it gives the advantage of easier detection of frontal and maxillary bone fractures as well as their displacement. The coronal reconstructed images have a superior role in the detection of the maxillary and orbital fractures. 3D images have a limited role in fracture involving the naso-orbital, naso-ethmoid region, and also when there is minimal fracture displacement. Though CT is a timeconsuming technique, it becomes superior or higher radiological investigation for better treatment outcome [43].

In mandibular CT imaging by particular MDCT, in comparison to Xray, It has been demonstrated to have higher sensitivity in detecting fractures of the mandible, and it provides excellent detail in detecting fractured mandibular condyle. This is due to high $\mathrm{z}$-axis resolution and detailed sagittal and coronal reconstructions that have a minimal artifact. In addition, the benefits of a 3D-CT reconstructed view, the spatial information provided by MDCT allows evaluation of severity and classification of the fracture. This can also be achieved with an OPG (Orthopantomogram) but requires a high technically skilled radiographer and an ambulant patient [43].

\section{CONCLUSION}

MDCT with MPR and 3D images turned into a standard part of the evaluation of maxillofacial damage on the account of the perfect affectability of this imaging system for fracture. In this appraisal, we talk in brief about the maxillofacial fractures utilizing MDCT, principally mid-facial fractures including maxillary fractures and mandibular fractures. As regards to the morphology of the fracture, the maxillofacial trauma is often complex, and the sustenance function of maxillofacial bones, including, smelling, breathing, speaking, seeing, and eating. Consequently, the precise diagnosis of maxillofacial fractures needs radiologic diagnosis through the utilization of MDCT and surgical 
management to preclude extreme restorative and utilitarian debilities.

\section{REFERENCES}

1. Mussa Q.H, Gatta I.S. The diagnostic value of computerized tomography in evaluation of maxillofacial trauma. Kufa.Med J 2009;(12):251-255.

2. Prasad V N, Khanal A. Computed Tomography evaluation of maxillofacial injuries. J. College of Medical Sciences Nepal. 2016;12(4):131-6.

3. Chalkoo AH, Nazir N, Yaqub G, Bhat $\mathrm{Z}$, et al. Incidence and patterns of fractures of Mandible, Midface or both using CT scan in trauma patients. International Journal of Oral Health Dentistry; 2017;3(1):29-33.

4. Adeel M, Rajput MS, Akhter S, et al. Anatomical variations of nose and paranasal sinuses; CT scan review. J Pak Med Assoc 2013;63(3):317-9.

5. Cashman EC, MacMahon PJ, Smyth D. Computed tomography scans of paranasal sinuses before functional endoscopic sinus surgery. World J Radiol 2011; 3:199-204.

6. Tan HM, Chong VF. CT of the paranasal sinuses: normal anatomy, variants, and pathology. CME Radiology 2001;2:120-5.

7. Ca glayan F, Tozo glu U. Incidental findings in the maxillofacial region detected by cone-beam CT. Diagn Interv Radiol 2012; 18(2):159-63.

8. Saigal K, Winokur RS, Finden S, et al. Useof three-dimensi0nal computerized tomography reconstruction in complex facial trauma. Facial Plast Surg 2005;21(3):214-20.

9. Ondik MP, Lipinski L, Dezfoli S, et al. The treatment of nasal fractures: a changing paradigm. Arch Facial Plast Surg 2009; 11(5):296-302.

10. Rodriguez ED, Stanwix MG, Nam AJ, et al. Twentysix year experience treating frontal sinus fractures: a novel algorithm based on anatomical fracture pattern and failure of conventional techniques. Plast Reconstr Surg 2008;122(6):1850-66.

11. Tiwari $\mathrm{P}$, Higuera $\mathrm{S}$, Thornton $\mathrm{J}$, et al. The management of frontal sinus fractures. J Oral Maxillofac Surg 2005;63(9):1354-60.

12. Obuekwe O, Owotade F, Osaiyuwu O. Etiology and pattern of zygomatic complex fractures: a retrospective study. J Natl Med Assoc 2005;97:992.

13. Martini JJ. Maxillofacial radiography. oral Surg Oral Med Oral Pathol 1950;3:1540-1556.

14. Andreasen Jo. Etiology and pathogenesis of traumatic dental injuries. A clinical study of 1,298 cases. Scand J Dent Res 1970;78:329342.

15. Moilanen A. Primary radiographic diagnosis of fractures in the mandible. Int J Oral Surg 1982;11:299-303.

16. Stacey DH, Doyle JF, Mount DL, et al. Management of mandible fractures. Plast Reconstructive Surg.2006; 117: 48-60.

17. Laine FJ, Conway WF, Laskin DM. Radiology of maxillofacial trauma. Curr Probl Diagn Radiol 1993; 22:145-188.

18. Seibert JA, Boone JM. X-ray imaging physics for nuclear medicine technologists, part 2: x-ray interactions and image formation. $\mathrm{J}$ Nucl Med Technol. 2005;33:3-18.

19. Goldman LW. Principles of CT: Multislice CT. Journal of Nuclear Medicine Technology. Vol. 36, No.2, 2008.

20. Semnic R. CT Toraksa i Abdomena, Institut za onkologiju Vojvodine, Sremska Kamenica, Grpah Style, Novi Sad 2005.

21. Stankovic JB, Milosevic NT. Osnovi radioloske fizike (Basic Principles of Radiological Physics), PTT, Belgrade, 2007.

22. Herman, G. T., Fundamentals of computerized tomography: Image reconstruction from projection, 2nd edition, Springer, 2009.

23. Berrington de González A, Mahesh M, Kim KP, Bhargavan M, Lewis R, Mettler F, Land C. "Projected cancer risks from computed tomographic scans performed in the United States in 2007". Arch. Intern. Med. 2009; 169 (22): 2071-7.

24. Brenner DJ, Hall EJ. "Computed tomography - an increasing source of 
radiation exposure" (PDF). N. Engl. J. Med., 2007; 357 (22): 2277-84.

25. Matsumoto K, Sawada K, Kameoka S, Yonehara Y, Honda K. Cone-beam computed tomography for the diagnosis of mandibular condylar fractures: 11 case reports. oral Radiol 2013;29:80-6.

26. Schuknecht B, Graetz K. Radiologic assessment of maxillofacial, mandibular, and skull base trauma. Eur Radiol 2005;15:560—8.

27. Salonen EM, Koivikko MP, Koskinen SK. Violence-related facial trauma: analysis of multidetector computed tomography findings of 727 patients. Dentomaxillofacial Radiol 2010;39:107-12.

28. Ogura I, Sasaki Y, Kaneda T. Analysis of mandibular condylar and glenoid fossa fractures with computed tomography. Eur Radiol 2014;24:902-6.

29. Escott EJ, Branstetter BF. Incidence and characterization of unifocal mandible fractures on CT. Am J Neuroradiol 2008;29:890-4.

30. Zhou HH, Liu Q, Cheng G, Li ZB. Etiology, pattern, and treatment of mandibular condylar fractures in 549 patients: a 22-year retrospective study. J Craniomaxillofacial Surg 2013;41:34-41.

31. Sawazaki R, Lima Jr SM, Asprino L, Moreira RWF, de Moraes M. Incidence and patterns of mandibular condyle fractures. J Oral Maxillofac Surg 2010;68:1252-9.

32. Lieger O, Zix J, Kruse A, lizuka T. Dental injuries in association with facial fractures. J Oral Maxillofac Surg 2009;67:1680 — 4.

33. Iida S, Kogo M, Sugiura T, Mima T, Matsuya T. Retrospective analysis of 1502 patients with facial fractures. Int $\mathrm{J}$ oral Maxillofacial Surg 2001;30:286-90.

34. Al Ahmed HE, Jaber MA, Fanas SHA, Karas M. The pattern of maxillofacial fractures in Sharjah, United Arab Emirates: a review of 230 cases. oral Surg Oral Med Oral Pathol Oral Radiol Endod 2004;98:166-70.
35. Yamamoto K, Kuraki M, Kurihara M, Matsusue Y, Murakami K, Horita S, et al. Maxillofacial fractures resulting from falls. J oral Maxillofacial Surg 2010;68:1602-7.

36. Sohns JM, Staab W, Sohns C, Schwarz A, Streit U, Hosseini AS, et al. Current perspective of multidetector computed tomography (MDCT) in patients after midface and craniofacial trauma. Clin Imaging 2013;37:728-33.

37. Smith HL, Chrischilles E, Janus TJ, Sidwell RA, Ramirez M, Peek- Asa C, et al. Clinical indicators of midface fracture in patients with trauma. Dent Traumatol 2013;29:313-8.

38. Yamamoto K, Matsusue Y, Murakami K, Horita S, Matsubara Y, Sugiura T, et al. Maxillofacial fractures due to work-related accidents. J Crani0maxillofac Surg 2011;39:1826.

39. Little SC, Kesser BW. Radiographic classification of temporal bone fractures: clinical predictability using a new system. Arch Otolaryngol Head Neck Surg 2006;132:1300-4.

40. Chu ZG, Yang ZG, Dong ZH, Chen TW, Zhu ZY, Deng W, et al. Features of craniomaxillofacial trauma in the massive Sichuan earthquake: analysis of 221 cases with multi-detector row CT. J Craniomaxillofac Surg 2011;39:503-8.

41. Schulze D, Heiland M, Thurmann H, Adam G. Radiation exposure during midfacial imaging using 4- and 16slice computed tomography, conebeam computed tomography systems and conventional radiography. Dentomaxillofacial Radiol 2004;33:83-6.

42. Kaeppler G, Cornelius CP, Ehrenfeld M, Mast G. Diagnostic efficacy of cone-beam computed tomography for mandibular fractures. oral Surg Oral Med Oral Pathol Oral Radiol 2013;116:98-104.

43. Sasaki Y, Kaneda T. Multidetector computed tomography of maxillofacial fractures. Japanese Dental Science Review 2014;(50): 86-90. 